\title{
Eva Stubenrauch
}

\section{Mahnung, Distanz, Resignifizierung}

\section{Textverfahren der Digitalisierungskritik}

Das Schreiben über gegenwärtige Netzwerkphänomene wird häufig von einer normativen Spur begleitet. Zu diesem Eindruck kommt, wer sich auch nur wenige Minuten mit aktuellen Zeitdiagnosen und Narrativen des Digitalen als mittlerweile unverzichtbarer Bestandteil unserer Gegenwartsgesellschaft auseinandersetzt. Das Netz erscheint als die passende Metapher für eine vielgestaltig diagnostizierte posthistorische Zeit, die nicht mehr nach vorn rückt, sondern grenzenlos in die Breite fließt - und als willkommener Anlass, eben diese Zeittendenz kritisch zu beäugen. Kaum eine Zeitdiagnose unserer Gegenwart, die ihre Beobachtungen nicht an digitalen Phänomenen und Entwicklungen festmachen will. Selbst die wissenschaftliche Beschäftigung mit Netzwerkphänomenen ist nicht frei von emotionalen Einstellungen zum eigenen Objektbereich. Alexander Friedrich formuliert das „Verhältnis von Metaphorizität und Normativität“ angesichts der Forschungslage zu Netz(werk)metaphern als bleibende „offene Frage“. ${ }^{1}$ Aus der scheinbar wertfreien Beschreibung des Netzes als Organisationsprinzip mit „dezentralen oder verteilten Strukturen“ wird so schnell die Utopie eines möglichen kollektiven „Ausbrechens des Denkens aus der vermeintlichen Linearität von Sprache und Schrift“. ${ }^{2}$ Diese Heilserwartung wird andernorts als naives „Klischee von Mütterlichkeit“ netzwerkstruktureller Eigenschaften entlarvt, als „,äußerst ideologische, illusionäre Vorstellung“, die die Kosten der Vernetzung nicht mitbedenke und ,realitätsinkongruent' die Netz-Konsequenzen der Verflachung ausblende. ${ }^{3}$

Auf der affirmativen Seite fallen dabei Deskription und Präskription zusammen, wird ,Vernetzung، oftmals zum emphatischen Programm, das es möglichst bald und auf vielen Ebenen umzusetzen gilt. ${ }^{4}$ Auf der pejorativen Seite wird die Deskription vielfach unterlaufen, lässt sich die Zeit-,Diagnose' nicht ohne den

1 Alexander Friedrich: Metaphorologie der Vernetzung. Zur Theorie kultureller Leitmetaphern. Paderborn 2015, S. 25.

2 Jörn Münkner/Jürgen Fröhlich: „Einleitung“. In: Netzstrukturen. Zur Kulturgeschichte sprachlicher, visueller und technischer Netze. Perspecuitas Internet-Periodicum für mediävistische Sprach-, Literatur- und Kulturwissenschaft (2005), S. 1-8, hier S. 4 f. http://www.perspicuitas. uni-essen.de/sammelbd/netzstrukt/einleitung.pdf [zuletzt eingesehen am 7.6.2021].

3 Gerhard Fröhlich: „Netz-Euphorien. Zur Kritik digitaler und sozialer Netz(werk)metaphern“. In: Alfred Schramm (Hg.): Philosophie in Österreich 1996. Wien 1996, S. 292-306, hier S. 303.

4 Vgl. Stefan Kaufmann: „Netzwerk“. In: Ulrich Bröckling u. a. (Hg.): Glossar der Gegenwart. Frankfurt a.M. 2004, S. 182-189, hier S. 182.

Ә Open Access. (C) 2022 Eva Stubenrauch, publiziert von De Gruyter. (c) BY-NC-ND Dieses Werk ist lizenziert unter einer Creative Commons Namensnennung - Nicht-kommerziell - Keine Bearbeitung 4.0 International Lizenz. https://doi.org/10.1515/9783110758603-004 
Krankheitskontext lesen, der ihrer historischen Semantik innewohnt. ${ }^{5}$ Geht man von der Ebene der Diskurssemantik weg und blickt auf die Verfahrensweisen des Schreibens und damit auf die Machart der Aussagen, so findet sich eine wenig überraschende quantitative Asymmetrie: Netzwerkstrukturelle Formen sind in der Textperformanz digitalisierungsemphatischer und -kritischer Statements nicht gleich verbreitet. Einer Ästhetik des digitalen Schreibens bedienen sich vermehrt Autor*innen, die in der netzmedialen Rahmung Chancen für die Textproduktion erkennen. ${ }^{6}$ Formästhetiken des Digitalen sind somit vor allem in solchen Texten zu finden, die dem Digitalen einen avantgardistischen Beitrag zur Innovation ,herkömmlicher‘ Textverfahren zusprechen und den Neuen Medien somit einen Mehrwert für ein Neues Schreiben abgewinnen. ${ }^{7}$ Netzwerkpoetiken profitieren hier von dem erzählerischen Potenzial neuer technischer Operationen, die über ihren Neuheitswert hinaus in steter Entwicklung begriffen sind und so ständig neue Verfahrensweisen produzieren. ${ }^{8}$

Weitaus weniger verbreitet und weniger besprochen ist der Umgang dezidiert digitalisierungskritischer Texte mit netzwerkstrukturellen Schreibweisen. Dass sich die Digitalisierungskritik Verfahrensweisen des Digitalen bedient, scheint zunächst als kontraintuitive Annahme - bis man genauer hinsieht. Die Asymmetrie in Vorkommen und Beachtung digitalisierungskritischer Netzwerkästhetiken bildet den Ausgangspunkt meiner folgenden Überlegungen. Dieser Beitrag rückt gerade jene zeitdiagnostischen Umgangsweisen mit Netzverfahren ins Zentrum, die in Anschlag gebracht werden, um vor einer zunehmenden Digitalisierung der Gegenwartsgesellschaft zu warnen. Mit ,Mahnung“, ,Distanz‘ und ,Resignifizierung‘ schlägt dieser Beitrag eine dreiteilige Systematik der Funktionen digitalisierungskritischer Schreibverfahren vor, die freilich korrigiert und erweitert werden

5 Vgl. zum begriffsgeschichtlichen Abriss der ,Zeitdiagnose“ Eva Stubenrauch: „Kontrapunkt moderner Historizität. Erschöpfung als Gegenwartsdiagnose bei Görres, Nietzsche und Gumbrecht“. In: Jan Gerstner/Julian Osthues (Hg.): Erschöpfungsgeschichten. Kehrseiten und Kontrapunkte der Moderne. München 2021, S. 27-48.

6 So betont etwa Berit Glanz, selbst Autorin im Internet, in ihrem Nachwort zu Sarah Bergers Kurztextsammlung bitte öffnet den Vorhang: @milch_honig 2019-2009 (2020) die besondere Entsprechung der Rezeptionsmodi sozialer Netzwerke mit Erzählverfahren der Autofiktion. Berit Glanz: „,Die abgeschnittene Person“ - Autofiktion in den sozialen Medien“. In: 54books. https://www.54books.de/die-abgeschnittene-person-autofiktion-in-den-sozialen-medien/, 04.03.2020 [zuletzt eingesehen am 7.6.2021].

7 Vgl. Steffen Martus/Carlos Spoerhase: „Gelesene Literatur in der Gegenwart“. In: Dies. (Hg.): Gelesene Literatur. Populäre Lektüre im Medienwandel. Sonderband Text + Kritik. München 2018, S. 7-20.

8 Vgl. für einen konzeptionellen Ansatz und einen genealogischen Überblick die Studie von Szilvia Gellai: Netzwerkpoetiken in der Gegenwartsliteratur. Stuttgart 2018, bes. S. 1-27. 
kann, hier aber einen ersten Vorstoß bildet, sich dem Phänomenbereich der digitalisierungskritischen Zeitdiagnose zu nähern.

Unter Netzwerkverfahren verstehe ich im Folgenden textstrukturelle Anordnungen, die eine netz(werk)artige Verflechtung imitieren und sich mit Netz(werk)metaphern beschreiben lassen. Auf Objekt- und Beschreibungsebene dominiert hier die Auffassung einer zeichenvermittelten Wirklichkeit, die als ein interkonnektives „Geflecht von Linien und Knoten, von Kanälen und Kreuzungen“ organisiert ist. ${ }^{9}$ Textuelle Netzwelten sind Konstruktionen aus untergründig weit verzweigten und dezentrierten Zusammenhängen, die eine „flach hierarchisierte, modular angeordnete, kommunikativ dicht gekoppelte Matrix“ bilden ${ }^{10}$ und deren Bindeglied weder eine Essenz noch eine Hierarchie, sondern die Relation ist. ${ }^{11}$ Eine netzwerkartige Textgenese lässt sich potenziell unabhängig von den besprochenen Inhalten in der Organisationsstruktur des Textes lesen und verfährt zunehmend ausdifferenzierend und selbstbezüglich. ${ }^{12}$

Weil auch das (Inter-)Textparadigma des ausgehenden 20. Jahrhunderts Beschreibungsgewohnheiten ausgebildet hat, mit denen semiotische Suprasysteme als Verwebungen und Verflechtungen gefasst werden, sind hier zwei weitere Spezifizierungen netzwerkartiger Schreibverfahren seit der Digitalisierung zentral. Erstens: Sie imitieren und inszenieren netzmediale Eigenheiten, etwa in der Begrenzung, Positionierung und Anordnung von Zeichen in Chatverläufen oder in der paratextuellen Aufbereitung konkret digitaler Materialität wie die der Siebensegmentanzeige. Zweitens: Schreibverfahren nach der Digitalisierung nehmen wie ihr diskurssemantisches Äquivalent performativ Stellung zu digitalen Phänomenen der Gegenwartsgesellschaft, etwa zu Formen der Bedeutungsgenese, Vergemeinschaftung oder algorithmischen Organisation von Wissen, ${ }^{13}$ und verhandeln in ihrer Form sowohl die Bedingungen der Möglichkeit subjektiven Eingreifens als auch die spezifische Zeitlichkeit einer digitalisierten Kultur.

Die vorgeschlagene dreiteilige Taxonomie betitelt mit ,Mahnung“, ,Distanz‘ und ,Resignifizierung` netzwerkstrukturelle Schreibverfahren der Digita-

9 Kaufmann: „Netzwerk“, S. 182.

10 Stefan Kaufmann: „Einleitung. Netzwerk - Methode, Organisationsmuster, antiessentialistisches Konzept, Metapher der Gegenwartsgesellschaft“. In: Ders. (Hg.): Vernetzte Steuerung: Soziale Prozesse im Zeitalter technischer Netzwerke. Zürich 2007, S. 7-21, hier S. 7.

11 Vgl. Kaufmann: „Netzwerk“, S. 184.

12 Siehe dazu Hartmut Böhme: „Einführung: Netzwerke. Zur Theorie und Geschichte einer Konstruktion“. In: Ders. u. a. (Hg.): Netzwerke. Eine Kulturtechnik der Moderne. Köln u. a. 2004, S. 17-36, hier S. 23 u. 33.

13 Vgl. zu diesen Merkmalen einer digitalen Kultur Felix Stalder: Kultur der Digitalität. Berlin 2016, S. 95-202. 
lisierungskritik, denen die Eigenschaft zukommt, dass sich ihre Form nicht von ihrer Funktion lösen lässt. Wenn eine Digitalisierungskritik mahnt, wenn sie sich distanziert oder wenn sie resignifiziert, dann auf eine jeweils eigene Machart, der die Illokution inhärent ist. Die Form ist mit ihrer Pragmatik durchzogen. Die Pragmatik erlaubt dann auch eine vergleichende Subsumption unterschiedlicher Formen in dieselbe Funktion der kritischen Schreibweise, sodass etwa Juli Zehs und Hans Ulrich Gumbrechts Texte trotz ihrer immensen Formdifferenz unter dieselbe Funktion - ,Distanz ‘ fallen. Solche Schreibweisen der Digitalisierungskritik, die ihrerseits netzwerkstrukturell verfahren, finden sich in den hier besprochenen Texten sowohl auf mikrostruktureller Ebene, in Form sich wiederholender digitaler Textpartikel im Fortlaufen von Handlung bzw. Argumentation, als auch auf makrostruktureller Ebene und damit als übergreifendes Organisationsprinzip des Textes. Die vorgeschlagene Taxonomie - Mahnung/Distanz/Resignifizierung - ermöglicht einen vergleichenden Zugriff auf die verschiedenen Formen der Digitalisierungskritik und ist im Folgenden exemplarisch zu entfalten. Mit ihrer Hilfe lässt sich am Ende, so der Anspruch, genauer bestimmen, wie die Digitalisierungskritik der Gegenwart operiert und warum sie sich solchermaßen intensiv der Formen bedient, von denen sie sich gleichzeitig entschieden abwenden möchte.

\section{Mahnung}

Unter Schreibverfahren der Mahnung fallen solche Textphänomene, die sich einer Netzwerkästhetik bedienen, um deren Problematik hervorzuheben. Die Netzstruktur wird nicht affirmativ im Sinne progressiver Stiltendenzen verwendet und auch nicht als Ausdruck seismographischer Gegenwartsprotokollierung, sondern sie wird mit einem warnenden Gestus kombiniert. Die Oberflächenstruktur mahnend verwendeter Schreibverfahren der Vernetzung unterscheidet sich also in vielen Fällen nur marginal von affirmativen Umformungen tradierter Darstellungsweisen. Der Unterschied liegt in der Motivation der Verwendung, die bei den einen in der technisch inspirierten Erweiterung des schriftstellerischen Formrepertoires, bei den anderen in der ideologiekritischen Analyse netzwerkstruktureller Phänomene und ihrer ubiquitären Wirkung zu suchen ist. Schreibverfahren der Mahnung verwenden die Zitation der Netzstruktur zur Aufklärung über die Gefahren der Digitalisierung. Dem kritisch-engagierten Impetus liegt dabei häufig die Annahme zugrunde, dass die mediale Durchdringung einer Gesellschaft besonders dann problematisch wird, wenn sie nicht (mehr) bemerkt wird. Wenn eine Gewöhnung eingetreten ist, dient die mahnende Sichtbarma- 
chung im Text zugleich der Transparenz eben jener Prozesse, die mehr oder weniger unbemerkt unseren Alltag durchdringen.

\section{Tobias Elsässer: Play}

Exakt diese Wirkung beansprucht Tobias Elsässers Jugendbuch Play (2020). Schon das Cover implementiert eine Text-Bild-Kongruenz und erweckt den Eindruck, ${ }^{14}$ der Roman handele vom gläsernen Menschen (vgl. Abb. 1). Dargestellt ist ein

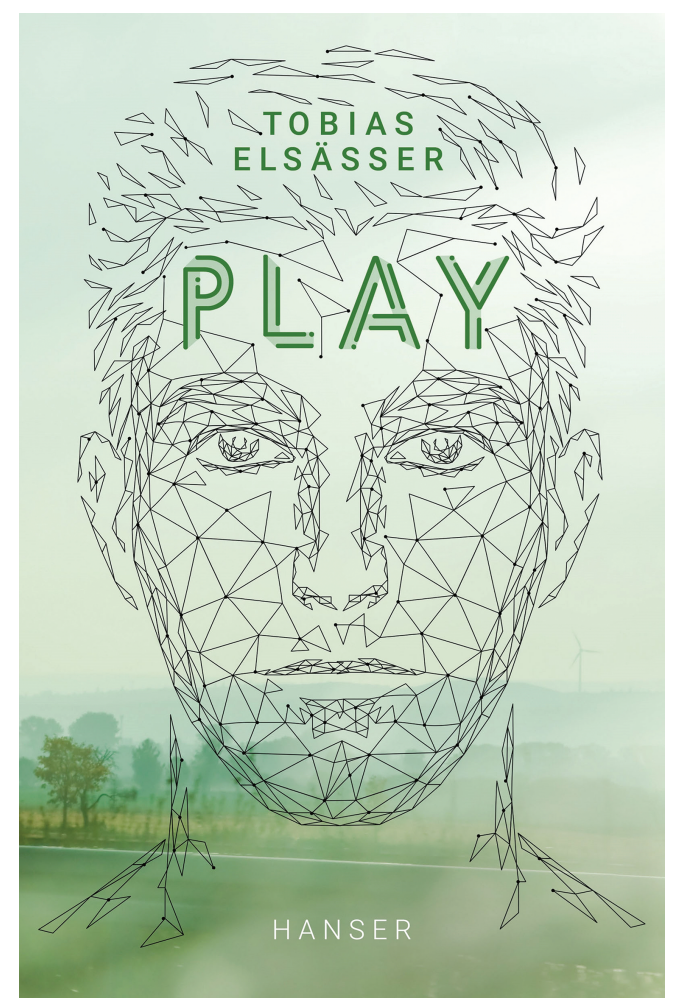

Abbildung 1: Cover von Tobias Elsässer: Play (2020).

14 Das Cover ist auf der Vertriebsseite des Hanser-Verlages einsehbar: https://www.hanserliteraturverlage.de/buch/play/978-3-446-26803-6/ [zuletzt eingesehen am 7.6.2021]. Copyright: Tobias Elsäßer, Play @ 2020 Carl Hanser Verlag GmbH \& Co. KG, München (Abdruck mit freundlicher Genehmigung des Carl Hanser Verlags). 
männliches Gesicht in Form eines Polygonnetzes, eines Gittermodells, das die computergrafische Basis für dreidimensionale Objekte bildet. Das Drahtgittermodell funktioniert als Grundlegung für Texturen, die die Struktur aus Kanten und Knoten mit Oberflächen füllen; je mehr Verzweigungen das Modell aufweist - wie hier an der Augenpartie angedeutet -, desto präziser, feiner und ,echter ${ }^{\star}$ wird das dreidimensionale Bild. Je höher die Anzahl der Polygone, desto mehr Rechenleistung wird für die Visualisierung des Modells benötigt. Das Cover spielt mit der visualisierten Metapher des vernetzten Menschen und nutzt eine Ästhetik der technologischen Berechenbarkeit des Individuums, ${ }^{15}$ die auf Handlungsebene aufgegriffen wird - geht es doch um die dystopische Vorstellung, eine App könne aus ungeschützten Daten sozialer Netzwerke präzise Modellierungen und Aussagen über das Selbst erstellen. Insofern die Covergrafik nicht das dreidimensionale Objekt mitsamt seiner Oberflächenstruktur, sondern lediglich die modellierende Grundmasse abbildet, wird die Aufmerksamkeit auf den technologischen Herstellungsprozess gelenkt. Der visuelle Paratext reflektiert damit die ,Gemachtheit ${ }^{\star}$ der Grafik, die in der ,authentischen“ Endversion des 3D-Objekts zu verschwinden droht und hier gerade der reflektierten Wahrnehmung überantwortet werden soll. In der intermedialen Formanleihe verweist die Medialität des Textes auf die Medialität von Big Data, deren Konstruktionsanteil bei der Datengenerierung an die Oberfläche geholt werden soll.

Wie ein Manifest gegen die „Materialvergessenheit“16 der digitalisierten Gegenwart zeigen sich Text und Paratext auch nach dem Aufschlagen des Buches: Die Kapitel sind mit segmentierten, dreidimensionalen Zahlen nummeriert, die den visuellen Stil der Titelgebung reproduzieren. Die Titelgebung wiederum verweist auf den Play-Button der App MASCHINE, durch dessen zweimaliges Betätigen die Handlung beginnt: ${ }^{17}$ Der 18-jährige Protagonist Jonas stößt kurz vor seinem Abitur online auf die MASCHINE, die vorgibt, seine Zukunft aus ihr verfügbaren Dateien berechnen zu können. Das Ergebnis - die Aussicht auf ein durchschnittliches Unternehmerleben wie das seines abwesenden Vaters - motiviert Jonas dazu, das Spiel ein zweites Mal zu beginnen und sich nach seinem Abitur auf einen Roadtrip zu begeben, der mit maximal unvorhergesehenen Handlungsverläufen die Berechnung der MASCHINE sabotieren soll.

15 Vgl. Martin Warnke: „Ästhetik des Digitalen. Das Digitale und die Berechenbarkeit“. In: Zeitschrift für Ästhetik und allgemeine Kunstwissenschaft 59 (2014), S. 278-286, hier bes. S. 279. 16 Siehe dazu grundlegend Stephan Kammer: „Visualität und Materialität der Literatur“. In: Claudia Benthien/Brigitte Weingart (Hg.): Handbuch Literatur \& visuelle Kultur. Berlin/Boston 2014, S. 31-47, S. 33.

17 Vgl. Tobias Elsässer: Play. München 2020, Prolog, o.S. 
Play verhandelt damit tradierte Topoi des Jugendromans - Reise, Selbstfindung, Distanzierung von den Eltern - im Gewand einer Digitalisierungskritik, die den Spannungsaufbau der Handlung aus einem steten Ankämpfen gegen den Algorithmus generiert. Dazu nutzt die Textoberfläche wiederholt grafische Abweichungen vom Fließtext in Form von Chatverlaufsabbildungen und Entscheidungsbäumen, die verzweigte Variablen auflisten und zwischen Entscheidungskontingenz und doch wieder vorgezeichneten Varianten changieren: Selbstbestimmtheit gegen die App und ihre Prognose avanciert zum zentralen Konflikt der Handlung, die voranschreitet, indem Jonas die algorithmischen Berechnungen mal befolgt, mal bewusst gegen sie entscheidet - und damit jede Entscheidung digital determiniert trifft. Die Agency, die Jonas der MASCHINE durch sein Verhalten zugesteht, wird im Text selbst reflektiert, doch braucht es dazu eine andere Figur: „Wenn du die App als Gegner betrachtest, hat sie bereits gewonnen. Du setzt auf Rot, auf Leute wie mich, mit denen $\mathrm{du}$ eigentlich nichts $\mathrm{zu}$ tun haben willst, weil sie, wenn deine Theorie stimmt, Konflikte und Ärger bedeuten. Sinnvoller wäre es, auf dein Bauchgefühl zu hören. Das macht dich weniger berechenbar. “18 Handlungslogisch konsequent endet der Roman damit, dass sich die App selbst in ihrer subversiven Programmatik gegen kommerzielle Datensammler offenbart. Die Sabotage der digitalen Manipulation dupliziert sich auf der Ebene des Manipulationsobjekts, das auf einmal zum Komplizen wird. ${ }^{19}$ An dem illokutionären Sprechakt des Romans - Warnung - lässt sich mithin wenig Zweideutiges finden. Vielmehr wird eindeutig ausgesagt, dass sich Algorithmizität nicht umgehen lässt. Die Botschaft ist klar: Wer sich einmal auf sie eingelassen hat, unterliegt ihrer „totalen Vernetzung“, mit der man sich auch zunehmend „selbst strangulieren“ kann. ${ }^{20}$

Materialität, Intermedialität und Handlungslogik in Play gehen Hand in Hand und erzeugen ein Konglomerat an Textverfahren, die der Funktion ,Mahnung' folgen. Visuell, reflexiv und narrativ wird somit die panoptische Vision einer vollständig vernetzten Gegenwart aufgerufen, in der selbst der Versuch,

18 Ebd., S. 218.

19 Vgl. ebd., S. 279.

20 Michael Andritzky/Thomas Hauer: „Alles, was Netz ist“. In: Klaus Beyrer/Michael Andritzky (Hg.): Das Netz. Sinn und Sinnlichkeit vernetzter Systeme. Heidelberg 2002, S. 11-18, hier S. 18. Auch dieser Text ist ein Beleg für die oben formulierte Hypothese, dass eine Vielzahl an wissenschaftlichen Publikationen mit Objektivitätsanspruch ihre normative Rahmung der Thematik nicht zurückhalten kann. Der Aufsatz beginnt mit einer epistemischen Klassifikation verschiedener Kontexte des ,Netzes“ - „Natur und Leben“, „Verkehr“, „Kommunikation“ und driftet in seinem letzten Abschnitt in eine Globalisierungskritik, die mit einem Plädoyer für ,kleine überschaubare Netze‘ endet. 
die Berechnung zu manipulieren, algorithmisch präfiguriert ist. Nicht nur zieht Elsässers Roman seine Handlungsmotivation aus einem Roadtrip, der gegen die technologische Prognostik gerichtet ist, er durchflicht seine Textoberfläche bis zum Schluss auch mit grafischen Markern digitaler Allpräsenz. Die Netzwerkstruktur als metaphorischer Inbegriff des ,Dazwischen“ verhindert also ihrerseits eine insulare Zwischenposition jenseits von Knoten und Kanten, da diese immer wieder nur Teil des Netzes sein kann. ${ }^{21}$ Das digitalisierungskritische Verfahren imitiert somit eine Netzwerkästhetik, um ihre Gefahren in vehementer Plastizität vorzuführen.

\section{Uwe Tellkamp: Der Eisvogel}

Formal ganz anders, jedoch funktional äquivalent verfährt Uwe Tellkamps Roman Der Eisvogel (2005), der in der Forschung vor allem hinsichtlich der Motive Terror und Rechtskonservatismus oder diskursanalytisch auf die feuilletonistische Auseinandersetzung mit der politischen Haltung seines Autors befragt wurde. ${ }^{22}$ Seinen Verfahren der Digitalisierungskritik, hier Teil einer generellen Medienkritik, wurde bisher kaum Beachtung geschenkt. Sabrina Wagner beschreibt Tellkamps Protagonisten Wiggo zwar aufgrund seiner Aversion gegen elektronische Medien als jungen Mann, „der auf seltsame Weise aus der Zeit gefallen wirkt“; ${ }^{23}$ Kai Sina bemerkt die kulturkritische Ablehnung der „permanente[n] und unentrinnbare[n] Bestrahlung durch die Medien, vor allem aber die andauernde Konfrontation mit den Nachrichten in den Medien“24 - verschärft allerdings sein Augenmerk der eigenen Hierarchisierung gemäß auf letztere.

21 Vgl. zur Metaphorik des Netzes als Struktur des ,Dazwischen“ Sebastian Gießmann: Netze und Netzwerke. Archäologie einer Kulturtechnik 1740-1840. Bielefeld 2006, hier bes. S. 23.

22 Vgl. Kai Sina: „Das Haus an der Havel gegen den Schmutz der Moderne. Kulturkritik bei Uwe Tellkamp“. In: Ders../Ole Petras (Hg.): Kulturen der Kritik. Mediale Gegenwartsbeschreibungen zwischen Pop und Protest. Dresden 2011, S. 33-50; Maike Schmidt: „,Zurück zum hohen Ton‘? Uwe Tellkamps Der Eisvogel im Feuilleton“. In: Dies. (Hg.): Gegenwart des Konservativismus in Literatur, Literaturwissenschaft und Literaturkritik. Kiel 2013, S. 295-307; Sabrina Wagner: Aufklärer der Gegenwart. Politische Autorschaft zu Beginn des 21. Jahrhunderts - Juli Zeh, Ilja Trojanow, Uwe Tellkamp. Göttingen 2015, bes. S. 223-228; Eva Stubenrauch: „Die eigene Zeit hassen. Zeitdiagnostik als Maßstab kollaborativer Wertung zwischen Gegenwart und Zukunft (Der Fall Tellkamp/Eisvogel)“. In: Sven Bordach u.a. (Hg.): Zwischen Halbwertszeit und Überzeitlichkeit. Geschichte der Wertung literarischer Gegenwartsbezüge. Hannover 2021, S. 41-64.

23 Wagner: Aufklärer der Gegenwart, S. 224.

24 Sina: „Das Haus an der Havel gegen den Schmutz der Moderne“, S. 37. 
Die Darstellung der „Bestrahlung durch die Medien“ lohnt jedoch ebenfalls einen Blick, greift der Text doch wiederholt auf verschiedene Schreibverfahren zurück, um die mediale Prägung der Gegenwart in seine Narration einzubinden und sie doch von ihr abzugrenzen. Mehrere Stellen im Roman thematisieren Wiggos Streifzüge durch die Großstadt, bei denen sich der Duktus des Erzählens verändert und in die Beschreibung von Momentaufnahmen übergeht. Geschildert wird die Begegnung mit medialer Vermittlung, die als ,porendurchdringender Dunst, ein elektrisch knisterndes Arom“25 gefasst wird. Sinnfällig beschreibt der Text eine unfreiwillige kollektive Rezeptionssituation, die der Alltag in seiner Gegenwart bereithält:

\begin{abstract}
Die Menschen waren hellwach und sogen es mit geheimer Gier ein, niemand konnte ausweichen, las man die Zeitungen nicht mehr, schaltete die Fernseher und Radios, die Faxgeräte und Computer ab, so waren doch in den Schulen, Universitäten, Fabriken und Büros Menschen, die sie nicht abbestellt oder abgeschaltet hatten, in den Schaufenstern flimmerten Videowände, Nachrichten schäumten auf wie zu lange gekochter Brei, Regenbogenmagma, schnell erstarrt, schon splitternd an den Straßenrändern, vom zerschnittenen Licht zerschnittene Scheiben, Zeitungen rollten auf, walzten sich in den U-Bahn-Stationen, an Kiosken zu farbkastenbunten Jagdstrecken aus, Handys plärrten, Short Messages tickten über Displays, Börsenbänder über Info-Screens [... ${ }^{26}$
\end{abstract}

Die ausladende Syntax in hypotaktischer Manie, die hier auch nur gekürzt angeführt wird, liest sich wie eine tradierte literarische Großstadtbeschreibung, in der allerdings nicht Menschen, Gebäude und Verkehr den Eindruck von Überfülle erzeugen, sondern die Rezeption medial vermittelter Nachrichten an allen Ecken und Plätzen. Detailliert beschrieben wird eine abwechselnde Medienwahrnehmung, die die Grenzen des materialen Raums und auch die Grenzen leiblicher Anwesenheit überschreitet: Die Perzeption flimmernder Bildschirme - „vom zerschnittenen Licht zerschnittene Scheiben“ - und rasch aufeinanderfolgender Short Messages generiert eine Diffusion der Körper im Raum, ein unausweichliches Eingebundensein in die technologische Omnipräsenz, die sich bis zu gewaltsamer Aufdringlichkeit steigert. Flimmern, Schäumen, Splittern, Zerschneiden, Rollen, Walzen, Plärren, Ticken überantworten die Handlungsmacht eindeutig der medialen Beschallung; die Technologie-Kritik speist sich hier aus dem Textverfahren synästhetischer Verdichtung, die wie der Satz kein Ende nimmt und keine nicht-semiotisierte Raumbeschreibung erlaubt. Gleichzeitig baut die Szene an einem zunehmend pejorativ gerahmten Narrativ medienhistorischer Evolution von Zeitung bis Short Message, die den Untergang des

25 Uwe Tellkamp: Der Eisvogel. Frankfurt a.M. ${ }^{4} 2018$, S. 39.

26 Ebd. 
Abendlandes mit einer Prozesslogik menschlichen Kontrollverlusts angesichts technologischer Entwicklung entwirft.

Nicht nur die Perzeptionsbeschreibung erinnert an die Ästhetik der Klassischen Moderne, auch die druckgrafisch und strukturpositionell auffälligen Nachahmungen des Zappens, Aufploppens und Klickens fügen sich harmonisch in die Tradition literarischer Darstellung bzw. Kritik zunehmender Modernisierung ein:

+++ AOL Time Warner 116,34 +++ 12 Technologies 53,21+++ Yahoo 56,40 +++ Du, ich hab 'nen Freund. - Ja, sicher. Klar. Deshalb können wir doch trotzdem. - Nee, du, Ficken is nich. CNN: It's the news. Soldaten und Nomaden. Pu:pushLetItGroove. EU legt Norm für Trillerpfeifenkugeln fest. Diesen Weg. Auf den Höhn. Bin ich oft gegangen. Vöglein sangen. Lieder. Excuses for travellers. Suddenly everything fell out of place Suddenly everything Suddenly everything ... everything ... ${ }^{27}$

Gänzlich unvermittelt und unerklärt schiebt sich ein solcher Abschnitt in die Narration, die ansonsten von multiperspektivischen Abschnitten des zusammenhängenden Fließtexts getragen wird. Weder Ich-Erzähler noch Figuren nehmen zu der Formanleihe an Newstickern, Werbebannern, Chatverläufen und Radiomusik Stellung, so dass die Addition textuell imitierter intermedialer Verweise wie aus der Form gefallen wirkt. Unterstützt wird dieser Eindruck durch den kursivierten und eingerückten Schriftsatz, der den Abschnitt noch stärker vom Rest des Textes isoliert.

Das Unverbundene des Medienmischmaschs bildet im Roman ein klares Oppositionspaar mit konventionellen und nichttechnisierten Formen der Kommunikation wie dem Briefeschreiben, „per Hand mit Tinte auf Papier“. ${ }^{28}$ Die orthographisch inkorrekte Buchstabentilgung, die auf den konzeptionell mündlichen Stil des Chattens referiert - „hab `nen“, ,is nich“-, wird in Eisvogel dann auch Teil standardisierter Argumente der Technikkritik: „Ich hasse E-Mails, die Leute können sich überhaupt nicht mehr ausdrücken, einen richtigen Brief schreiben“. ${ }^{29}$ Der ,richtige Brief" nimmt außerdem eine andere Stellung als die

27 Ebd., S. 110, Herv. i. O.

28 Ebd., S. 114.

29 Ebd. Siehe zu diesen kulturkritischen Topoi der Warnung vor medialer Evolution Kathrin Passig: Standardsituationen der Technologiekritik. Berlin 2013, S. 9-25. Kai Sina weist zurecht darauf hin, dass der Roman seine rechtskonservativen bis -radikalen Äußerungen in die Figurenrede verlagert und in großen Teilen durch Kontrastierung mit anderen Figuren relativiert. Die Aussage Wiggos kann somit nicht mit der Aussage des Textes identifiziert werden. Sina macht jedoch ebenso folgerichtig auf den Umstand aufmerksam, dass die medienaversive Kulturkritik keine Gegenstimmen im Roman findet und entsprechend keine Distanz zu ihr aufgebaut wird. Der Eisvogel kann damit im Ganzen technologiekritisch gelesen werden. Vgl. Sina: „Das Haus an der Havel gegen den Schmutz der Moderne“, S. 46. 
Medienmimesis im Narrationsverlauf ein, wird zwar auch kursiviert wiedergegeben, aber eingebunden in Tagesablauf und erlebte Rede der Figuren, noch dazu hinsichtlich der Eigenarten der Handschrift kommentiert sowie als Textgattung „Brief“ klar benannt. ${ }^{30}$ Der handschriftliche Brief, so die Logik der Verfahrensweise, ist erzählerisch anschlussfähig, kann eingebunden und wiedergegeben werden, kann etwas bewirken und die Narration beeinflussen. Das Mediengemisch hingegen entzieht sich der klaren Einordnung, steht zu den Figuren in keinerlei reziproker Beziehung, sondern unterbricht die Kohärenz ihres In der Welt-Seins, und wirkt vermischend, verflachend und zersetzend auf den Fluss des Kommunikationsorgans Sprache ein. Es drängt sich auf, stört gewollte Insularität und Linearität mit zwanghafter Vernetzung und nivelliert Unterscheidungen, die sowohl der auf Aus- und Abgrenzung gepolte Plot des Romans als auch die rhetorische Reminiszenz an die Kulturkritik der Frankfurter Schule so dringlich anstrebt.

Mit Elsässers Roman teilt Tellkamps literarische Zeitdiagnose die Pragmatik der Mahnung, die die Verfahren beider Texte durchzieht: Beide nutzen Formanleihen an digitale Formate, um auf ihre Folgen für Individualität, Sozialität und Textproduktion aufmerksam zu machen; wie Play setzt auch Der Eisvogel dabei auf die Zitation netzwerkstruktureller Formsegmente, um sie und ihre störende Wirkung weitgehend unkommentiert der durch die Texthaltung kritisch präformierten Lektürehaltung zu überlassen. Im Unterschied zu Elsässer baut Tellkamp jedoch auf die klassisch konservative Unterscheidung zwischen E- und U-Kultur sowie von digitaler Vermittlung und scheinbarer analoger Unmittelbarkeit. Die Differenz auf der Verfahrensebene liegt darin, dass Play die totale digitale Durchdringung sichtbar macht, Tellkamps Roman hingegen aus der Opposition von medialer Berührung und Unberührtheit sein kulturkritisches Programm generiert; totale Sichtbarkeit und Reizüberflutung stehen hier im Kontext eines elegischen Verlustnarrativs, das die nostalgische Utopie vortechnisierter Kommunikation als Gegenmodell zur medialisierten Gegenwart aufruft. Obwohl zwei 15 Jahre auseinanderliegende Romane mit gänzlich verschiedenen Themen und Adressaten, produzieren doch beide gleichermaßen eingängig in ihrer Formgebung technologie- und netzkritische Verfahren, die die Mahnung als relevante Geste der digitalisierungsskeptischen Gegenwartsliteratur ausweist.

30 Vgl. Tellkamp: Der Eisvogel, S. 216 u. 218 f. 


\section{Distanz}

Kulturkritik im engeren Sinne braucht ein Verständnis von Historizität und hat ihre Wurzeln in der europäischen Aufklärung. Ist doch ihr Grundgestus, auf Basis eines selbstreflexiven Beobachtungsstandpunkts in der Gegenwart deren defizitären Status im Vergleich zu sentimentalisch idealisierten Vergangenheiten zu beklagen. ${ }^{31}$ Seit 1900 lässt sich darüber hinaus ein zunehmender Schwund der Zukunft als segensreiche Projektionsfolie für die Verbesserung gegenwärtiger Zustände beobachten, der auch in den Zeitdiagnosen des 21. Jahrhunderts und ihrer Kollektivthese einer deterministischen Gegenwart in Ausdehnung wiederholt wird.

\section{Hans Ulrich Gumbrecht: Unsere breite Gegenwart}

Unter zahlreichen Belegstellen für dieses räumlich dominierte Zeitgefühl nennt Hans Ulrich Gumbrecht auch die „Hyperkommunikation“, ${ }^{32}$ deren grenzenlose und unentrinnbare Netzwerkbildung „unsere breite Gegenwart“ versinnbildliche. ${ }^{33}$ Das Netzwerk ist hier Metapher für eine Zeitwahrnehmung, in der der Eindruck dominiert, „daß unsere Gegenwart sich verbreitert hat, da sie nun von einer Zukunft, die wir nicht mehr sehen, erreichen oder wählen und einer Vergangenheit, die wir nicht mehr hinter uns lassen können, umgeben ist“. ${ }^{34}$ Gumbrechts Text bringt gegen die seinen Autor bedrängenden „Zudringlichkeiten elektronischer Kommunikation“ in Form von „mehreren hundert E-Mails, die ich im Laufe eines normalen Arbeitstages erhalte“ ${ }^{35}$ ein anekdotisches Schreiben in Anschlag. Ohnehin durchzieht die Logik selbsterlebter Fallbeispiele für Inseln der Präsenz innerhalb des Stroms der breiten Gegenwart die Gesamtstruktur des zeitdiagnostischen Buches. Die Abkapselung von der „Hyperkommunikation“ im Schutzraum eines kleinen Büros innerhalb der Universitätsbibliothek ist in einer Reihe mit Zuschauersport und intensiven

31 Vgl. Georg Bollenbeck: „Kulturkritik: Ein unterschätzter Reflexionsmodus der Moderne“. In: LiLi 137 (2005), S. 41-53, hier S. 48.

32 Vgl. zur Analyse der Simultaneitätsdiagnose und zu ihrer Historisierung im Vergleich mit Nietzsches Lebensphilosophie Eckhard Schumacher: „Present Shock. Gegenwartsdiagnosen nach der Digitalisierung“. In: Merkur 72/826 (2018), S. 67-77, hier S. 74 f.

33 Ich beziehe mich hier auf das letzte ,Analysekapitel‘, „Unbegrenzte Verfügbarkeit. Über Hyperkommunikation (und Alter)“, in: Hans Ulrich Gumbrecht: Unsere breite Gegenwart. Übers. v. Frank Born. Berlin ${ }^{2} 2015$ [2010], S. 114-131.

34 Ebd., S. 49.

35 Ebd., S. 117. 
Erlebnissen zwischen Leser und literarischem Klassiker verortet. Gumbrecht baut somit schreibend ein Paradigma präsentischer Erfahrung auf, das ein Verhindern von Präsenz in der hektischen, digitalisierten und zeichenvermittelten Gegenwart als sein konstitutives Außen braucht.

Das anekdotische Schreiben unterstützt auf Form- und Strukturebene die programmatische Abschottung von der Zeit. Textpragmatisch dient die Reihung kurzer lebensweltlicher Ausschnitte dazu, sich mittels präsentischer Kompensation vom Außen zu distanzieren, und das auch in der Schreibweise, die auf die „sinnfällige Prägnanz“ der Anekdote setzt. ${ }^{36}$ Paradoxerweise funktioniert diese Distanz mit Nähe: Seine „,antielektronische Haltung،37 begründet Gumbrecht mit zahlreichen Verweisen auf sein fortgeschrittenes Alter und sein ,Altmodisch-Sein', ${ }^{38}$ das ihn zum Vergleich der digitalen und vordigitalen Zeit prädestiniert und noch dazu den Topos des unzeitgemäßen Zeitgenossen bedient. ${ }^{39}$ Diese Schreibhaltung durchzieht jedoch weniger eine meritokratische Distanzierung als vielmehr ein Gestus der Augenhöhe mit den Lesenden, die über die Geschichten des in die Jahre gekommenen Kulturkritikers des Öfteren zum Schmunzeln gebracht und - verstärkt durch den anekdotischen Modus des Beiläufigen - Verständnis für seine Digitalaversion ausbilden sollen. Statt als „elektronisch erreichbare[r] Jedermann“ 40 inszeniert sich Gumbrecht als digitaler Eremit, der sich seine ,Gesprächspartner' selbst aussucht und seine Lesenden gewollt ins Vertrauen zieht, indem er beispielsweise frei heraus, gesteht', noch immer handschriftlich Vorträge zu schreiben. ${ }^{41}$

Der Hyperkommunikation seiner Gegenwart wirft der Kritiker vor, dass sie „die Konturen zerfrißt, die bislang meinem Alltag Struktur und Spannung gegeben haben“. ${ }^{42}$ Er beklagt die abnehmende Ereignishaftigkeit kommunikativrhetorisch „harte[r] Übergänge“43 und betrauert die „Brüche und Grenzen“, die

36 Christian Moser: „Die supplementäre Wahrheit des Anekdotischen. Kleists ,Prinz Friedrich von Homburg' und die europäische Tradition anekdotischer Geschichtsschreibung“. In: KleistJahrbuch 2006. Hg. v. Günter Blamberger u. a. Stuttgart, Weimar 2006. S. 23-44, hier S. 23.

37 Gumbrecht: Unsere breite Gegenwart, S. 122.

38 Ebd., S. 131.

39 Vgl. Giorgio Agamben: „Was ist Zeitgenossenschaft?“ In: Ders.: Nacktheiten. Frankfurt a.M. 2010, S. 21-35.

40 Vgl. Gumbrecht: Unsere breite Gegenwart, S. 118.

41 Vgl. ebd., S. 126.

42 Ebd.

43 Ebd. 
auf dem „ewigen Computerbildschirm“ eingeebnet würden. ${ }^{44}$ Seine Diagnose „Alles schmilzt ineinander, alles ist ,fusion“““5 - belegt er mit mehreren anekdotischen Einblicken, wie die Schilderung eines denkwürdigen Erlebnisses auf dem täglichen Nachhauseweg exemplarisch zeigt:

Ich kann mich noch gut an den späten Nachmittag erinnern, als ich auf dem Weg nach Hause die Straße versperrt von den Büchern und Möbeln eines Kollegen vorfand, dessen Frau sie aus dem Fenster geworfen hatte nach der Lektüre der täglichen E-Mail an seine Geliebten, die sich beide nicht kannten - eine war eine Studentin, die andere eine Kollegin -: Es war eine E-Mail, die er aus (erstaunlichem) Versehen sowohl an seine Gattin als auch an die Kanzlerin der Universität geschickt hatte. ${ }^{46}$

Mit den „Gefahren des Ineinanderübergehens“ und dem durch sie verursachten „Hauch erotischer Aufladung“ erklärt sich Gumbrecht im Anschluss an die anekdotische Sequenz die Motivation seines Kollegen, für den Austausch mit den Geliebten auf elektronische Kommunikationsmittel zurückgegriffen zu haben. ${ }^{47}$ Die tragikomische Miniaturnarration setzt gegen die beklagte Nivellierung der Netzkommunikation eine „wahre, noch unbekannte, merkwürdige Begebenheit“ “ ${ }^{48}$ die ihre Pointe durch eine diskontinuierliche Abweichung von Gewohnheiten erhält. Der anekdotische Einschub impliziert Augenzeugenschaft sowie formale Kürze und führt im unerbittlichen Kommentar humoristisch die alles fusionierende Netzkommunikation mit dem faux pas einer Selbstentlarvung von Untreue eng.

In Gumbrechts Abhandlung hat die anekdotische Form eine klare Funktion: Sie führt die Grenzen und Brüche ein, die die hyperkommunikative Gegenwart nicht mehr kenne, insofern sie nicht nur vom Rahmenbruch erzählt, sondern selbst einen solchen darstellt. Sie markiert ein Moment des Unvorhergesehenen und der Nahkommunikation, bringt als Erzählung zwischen Fakt und Fiktion den Anspruch lebensweltlicher Beglaubigung in die Argumentation ein und wirkt durch die pointierte Fassung als „disruptive Kraft“, ${ }^{49}$ mit der die (Klage über die) Stagnation der Gegenwart durchbrochen wird. Das anekdotische Schreibverfahren distanziert sich von der Netzwerkstruktur, wie Gumbrecht sie versteht,

44 Ebd., S. 130.

45 Ebd.

46 Ebd., S. 123.

47 Ebd., S. $123 \mathrm{f}$.

48 Sonja Hilzinger: Anekdotisches Erzählen im Zeitalter der Aufklärung. Zum Struktur- und Funktionswandel der Gattung Anekdote in Historiographie, Publizistik und Literatur des 18. Jahrhunderts. Stuttgart 1997, S. 17.

49 Moser: „Die supplementäre Wahrheit des Anekdotischen“, S. 24. 
bringt es doch ein Mindestmaß an Anschaulichkeit und Verdichtung in die hyperkommunikative Zeit der ubiquitären losen Verbindungen. Gegen die diagnostizierte gähnend mittelmäßige und aufdringliche elektronische Kommunikation führt die textperformative Distanzierung mit dem Anekdotischen ein Verfahren ins Feld, das seinen epistemischen Wert aus Bildlichkeit, Dramatik und Witz bezieht. ${ }^{50}$

\section{Juli Zeh: Leere Herzen}

Schreibverfahren der Distanz zeichnen sich dadurch aus, dass sie in der Textgenese gegen eine selbstthematisierte Netzwerkstruktur prozessieren. Die performative Abgrenzung gegen die kritisierte Gegenwartstendenz ist hier das entscheidende Merkmal. In dem, wogegen angeschrieben wird, sind digitalisierungskritische Ansprüche häufig kongruent: Negativfolie ist meist die Nivellierung, Verflachung und Vereinnahmung digitaler Medien und der von ihnen verursachten Zeitwahrnehmung der Gegenwart. Ein Vergleich der Gegenmittel des Diagnostizierten fördert jedoch bemerkenswerte Unterschiede zutage. Die Abwehrhaltung ist demnach oft sehr ähnlich, die Schreibstrategie der Abwehr hingegen different. Wählte Gumbrecht mit anekdotischen Verfahren einen Modus der Nähe, Brüche und Kontingenz, so greift Juli Zeh in ihrem digitalisierungskritischen Roman Leere Herzen (2017) auf eine gänzlich andere Schreibweise zurück. Hier dominiert nicht das wiederholte pointierte Fragment, sondern ein linearer Spannungsaufbau, der an topische Strukturen der Heldenreise anschließt und der kritisierten Algorithmierung der Gegenwart einen narrativen Ausweg entgegenhält.

Obwohl in Romanform, handelt es sich bei Leere Herzen ebenfalls um eine Zeitdiagnose, die paratextuell angekündigt wird. „Da, so seid Ihr“, bildet das vorangestellte Motto der Handlung, die mit der Suizidpraxis „die Brücke“ und ihrem Angebot des sinnhaften Freitods für Individuen, deren suizidale Tendenz mittels des Algorithmus Lassie in Netz und Darknet ermittelt wird, eine deutlich nahzeitdystopische Qualität aufweist. ${ }^{51}$ Akteurnetzwerktheoretisch perspektiviert, erzählt der Roman von sozialen Netzwerkstrukturen, die der Algorithmus

50 Vgl. für diese Eigenschaften der Anekdote als literarische Form mit ästhetischer und epistemologischer Funktion Christian Moser: „Von der Sonne der Wahrheit zum Blitz der Erkenntnis: Epistemische Funktionen der Anekdote - Antike und Neuzeit im Vergleich“. In: Roland Ißler u. a. (Hg.): Europäische Gründungsmythen im Dialog der Literaturen. Bonn 2019, S. 463-476.

51 Vgl. Anne Fuchs: Precarious Times. Temporality and History in Modern German Culture. Ithaca 2019, S. 282-287. 
zwischen den als unzweifelhaft suizidbereit getesteten Individuen und politischen Organisationen - von Tierschutz bis Kalifat - generiert; Lassie hat damit eine zentrale handlungstragende Funktion. Algorithmizität prägt die pro- und antagonistischen Netzwerke, vermittelt zwischen ihnen und kulminiert schlussendlich in der Frage, ob private und politische Veränderung und damit eine alternative Zeitordnung möglich ist.

Neben der bei Juli Zeh erwartbaren Kritik an Datentransparenz hält die literarische Zeitdiagnose auch eine Medienkritik bereit: Der Text reißt wiederholt die ,Überschwemmung“ mit Informationen an, die zur Folge hat, „dass seit Jahren niemand mehr weiß, was er denken soll“. ${ }^{52}$ Die Kritik an der zunehmenden digitalen Überwachung und Informationsdichte der nahenden Gegenwart von 2025 ist in Zehs Roman jedoch nur auf semantischer Ebene präsent, während die Handlungsstruktur ein erzählerisches Gegenmittel anbietet: Die klare Distanz zwischen Netzwerkkritik und Netzwerkverfahren zeigt sich hier im makrostrukturellen Narrationsgang, der den Ausgangs- und Endpunkt der Handlung an denselben Ort, dieselbe Figurenkonstellation sowie dieselbe Rezeptionssituation bindet und auf Grundlage dieser Ähnlichkeit die Differenz in der Charakterentwicklung seiner Protagonistin Britta verortet: Der Anfang - „Knut und Janina kommen um fünf“53 - exponiert den Grundkonflikt der Handlung, indem die Fernsehnachrichten über ein Selbstmordattentat berichten, das nicht von Brittas Klienten verübt wurde und auf ein mit der Brücke konkurrierendes Terrornetzwerk hindeutet. Das Ende - „Knut und Janina kommen um sechs“"54 - schildert ebenfalls eine Rezeption der Fernsehnachrichten, die nun von einem Selbstmordattentat berichten, das nach außen wie ein missglückter Putschversuch des gegnerischen Terrornetzwerkes aussieht, tatsächlich aber eine Intervention der Brücke in den Putschversuch und damit eine Verteidigung der bestehenden Regierung im Modus konkurrierender Anschläge darstellt.

Anfangs- und Endszene verweisen aufeinander und machen in ihrer zirkulären Anlage auf den Reifeprozess der Hauptfigur aufmerksam: Die Narration funktioniert nach dem Schema der säkularisierten Heldenreise, wie sie auch in romantischen Reiseschilderungen topisch geworden ist: Trennung von der Welt, Durchkämpfen zu höherer Einsicht, Rückkehr an den Ursprungsort, nun aber mit der Kraft, die Mitmenschen mit der Einsicht zu segnen. ${ }^{55}$ Am gleichen Ort endend, an dem sie begonnen hat, ist Britta nicht nur von ihrer inneren Resignation geheilt,

52 Juli Zeh: Leere Herzen. München 2017, S. 20.

53 Ebd., S. 9.

54 Ebd., S. 332.

55 Vgl. Jonathan Campbell: Der Heros in tausend Gestalten. Übers. v. Karl Koehne. Berlin ${ }^{6} 2019$, S. 42-52. 
sondern wirkt zudem missionarisch auf ihre Freunde ein: „,Erst geht ihr jahrelang nicht wählen, und dann findet ihr es super, wenn das Regierungsviertel in die Luft gejagt wird?' Das betretene Schweigen dauert an. ${ }^{\text {“56 }}$ Leere Herzen verfolgt damit eine aufklärerische Mission: Die Einsicht, dass die Gegenwart falsch läuft, wird gepaart mit der Einsicht, selbst etwas an der eigenen und kollektiven Haltung zur Zeit ändern zu können - und damit trotz politischer Einwände mit der Regierung auch die Demokratie zu retten. ${ }^{57}$ Zehs Zeitdiagnose bringt also genau das in Anschlag, was digitalisierungskritischen Stimmen oftmals fehlt: Narrative Kohärenz, sichtbare zukunftszugewandte Sukzession, überschaubare Linearität und tiefenstrukturelle Entwicklung.

Hans Ulrich Gumbrecht und Juli Zeh setzen beide auf eine maximale Distanz zwischen den Inhalten ihrer Diagnose - breite Gegenwart, postpolitische Stagnation, digitalmediale Überschwemmung - und den Ausdrucksformen des Diagnostizierens. Bei Gumbrecht dominiert ein kompensatorischer Anspruch, der in einer Art Galgenhumor lediglich Strategien des Durchhaltens vorstellt. Bei Zeh wird ein narrativer Ausweg angeboten, der Subjekt und Umweltsituation in der Einsicht notwendigen politischen Engagements versöhnen soll. Das anekdotische Verfahren setzt damit auf Witz und persönliche Nähe, um ,angenehm' zu diagnostizieren; die narrative Zielführung der Heldenreise setzt stattdessen auf Kohärenz und Figurenentwicklung, um zur Überwindung der Lage aufzurufen. Die Diskurssemantik, derer sich beide Texte durch ihre zeitdiagnostische Pragmatik verpflichten, dient beiden als Sprungbrett, um ihr fehlendes Einverständnis mit der gegenwärtigen Lage durch stilistisch wiedererkennbare Merkmale ihrer Autorschaft auf Formebene zu positionieren.

\section{Resignifizierung}

Im Unterschied zu Verfahren der Mahnung, die eine Digitalästhetik reproduzieren, um ihre Allgegenwart zu kritisieren und auf ihre Folgen hinzuweisen, und zu Verfahren der Distanz, die den kritisierten Gegenwartserscheinungen ganz andere, kompensierende Formen entgegensetzen, folgen Verfahren der Resignifizierung

56 Zeh: Leere Herzen, S. 346.

57 Auf die immersionshemmenden Brüche in der klischeebesetzten Handlungslogik mit Blick auf das Ende verweist Sabine Schönfellner: „Erzählerische Distanzierung und scheinbare Zukünftigkeit. Die Auseinandersetzung mit biomedizinischer Normierung in Juli Zehs Romanen ,Corpus Delicti‘ und ,Leere Herzen‘. In: Zeitschrift für Germanistik 3 (2018), S. 540-554, hier S. 546. 
einem Anspruch des subversiven Zitats. Die textuelle Aufnahme von Netzwerkstrukturen hat hier - anders als in Verfahren der Mahnung - nicht das Ziel, durch Imitation verdeckte Wirkmechanismen zu entlarven, sondern dient dazu, aus der Übernahme des kritisierten Codes seine Neutralisierung herzustellen.

Das Konzept der Resignifizierung ist Teil der Performativitätstheorie Judith Butlers, die es wiederum in Auseinandersetzung vor allem mit John L. Austin und Jacques Derrida entwickelt hat. Austins Auffassung, die Gelingensbedingungen des performativen Sprechakts seien eminent an seinen Kontext gebunden, findet in Derrida einen Kritiker, der die performative Kraft vom Kontext in das Zeichen selbst verlagert: „Der ,Ritus“ ist keine [situative] Eventualität, sondern als Iterierbarkeit ein strukturelles Merkmal jedes Zeichens. "58 Zeichen erhalten laut Derrida eine performative Wirkung aus ihrer Iterierbarkeit, aus ihrer Lesbarkeit als wiederholendes Zitat einer konventionalisierten Handlung. Die Iterierbarkeit des Zeichens generiere dann auch die Möglichkeit des Bruches mit dem Kontext, woraus sich Bedeutungsverschiebungen des Zeichens in seiner Wiederholung ergeben und somit eine Dynamisierung des Zitats folgt. ${ }^{59}$ Auf diese Thesen aufbauend entwirft Butler eine Theorie subversiver Sprechakte, die sich gegen sprachliche Verletzung zur Wehr setzen, indem sie sich diese aneignen, und aus ihrer Zitation eine Wirklichkeitsveränderung herbeiführen: „Die Resignifizierung des Sprechens erfordert, daß wir uns neue Kontexte eröffnen, auf Weisen sprechen, die noch niemals legitimiert wurden, und damit neue und zukünftige Formen der Legitimation hervorbringen. “60 Die Verschiebbarkeit des Kontextes durch sprachliche Wiederholung erhält demnach ein revolutionäres Potenzial. Die widerständige Zitation bringt sprachliche Selbstreferenz, kontextuelle Kontingenz und zukunftsoffene Veränderbarkeit - Performativität per definitionem ${ }^{61}-z^{-}$sammen und verknüpft sie darüber hinaus mit der Möglichkeit, vergangene Verletzungen durch gegenwärtige Aneignung zu transformieren.

Zugegeben kontraintuitiv und beinahe makaber wirkt eine Übertragung des Konzepts der Resignifizierung auf Digitalisierungskritik, zielen Butlers Überlegungen doch vor allem auf eine Beschreibung der Selbstermächtigung vormals unterdrückter Gruppen. Strukturell und pragmatisch funktioniert der Mechanismus der letzten Verfahrenskategorie, die dieser Beitrag herausstellt, jedoch identisch: Resignifizierend sind Verfahren, die aus der zitathaften Wiederholung

58 Jacques Derrida: „Signatur Ereignis Kontext“. In: Ders.: Randgänge der Philosophie. Hg. v. Peter Engelmann, 2., überarbeitete Aufl. Wien 1999, S. 325-351, hier S. 343.

59 Vgl. Jörg Volbers: Performative Kultur. Wiesbaden 2014, S. 27.

60 Judith Butler: Hass spricht. Zur Politik des Performativen. Berlin ${ }^{5} 2016$, S. 71.

61 Siehe zur näheren Bestimmung des Performativen Erika Fischer-Lichte: Performativität. Eine Einführung. Bielefeld 2012, S. 68-72, hier S. 71. 
digitaler Phänomene ihre ,Reinigung` von bedrohlicher Wirkung oder Beschneidung menschlicher Agency herbeiführen. Auch hier impliziert die Resignifizierung, dass Kontexte verschoben werden und die sprachliche Anleihe aus der Referenz eine neue Pragmatik erzeugt. Nicht selten operieren auch digitalisierungskritische Verfahren der Resignifizierung mit dem Anspruch auf Selbstermächtigung und nutzen die kontextuelle Offenheit stilistischer Entlehnung, um im Zitat neue Zielsetzungen anzugehen und auf Grundlage der Wiedererkennbarkeit eine eigene Programmatik zu entwerfen.

\section{Roberto Simanowski: Stumme Medien}

Roberto Simanowskis „Streitschrift für eine neue Medienbildung“62 aus dem Jahr 2018 beginnt unvermittelt mit einer spekulativen Narration, in der von einem zukünftigen Standpunkt aus rückblickend die Präsidentschaft Mark Zuckerbergs als logische Nachfolge von Donald Trump motiviert wird. In einer mediengeschichtlichen Entwicklungslogik erzählt Simanowski den Aufstieg eines Mannes, der seinen Ruhm nicht länger aus Film und Fernsehen, sondern aus weltweit wirkenden sozialen Netzwerken bezog, um, so die verschwörungstheoretisch anmutende Erklärung, bewusst und heimlich seine Machtübernahme vorzubereiten und sein politisches „Programm der Visuellen Empathie (PVE)“ zu entwickeln, ${ }^{63}$ das sich zum Ziel setzt, durch die „Verschiebung der Kommunikation vom rational-sprachlichen Bereich in den emotional-visuellen und von den politischen Kontroversen zu den Freuden des Alltags“ den Menschen ,als sprachliches und politisches Wesen zu überwinden“. ${ }^{64}$ Über die Auflistung mehrerer öffentlicher Auftritte Zuckerbergs in den 2010er Jahren, die sich rückblickend als sukzessive Vorbereitung seiner Wahlkampagne lesen lassen, entwirft Simanowski mit einem an den Aufstieg des Facebook-Magnaten gekoppelten Modell der „globale[n], affirmative[n] Gemeinschaft" die Weltgesellschaft der nahen Zukunft. ${ }^{65}$

Dieser Bucheinstieg wird nach etwas mehr als zwei Textseiten abgebrochen und in die Frage überführt, ob „man so oder ähnlich irgendwann Mark Zuckerbergs Wahl zum Präsidenten der USA kommentieren“ werde. ${ }^{66}$ Die kausallogische Erzählung der nahenden politischen Weltlage bedient sich - das wird

62 Roberto Simanowski: Stumme Medien. Vom Verschwinden der Computer in Bildung und Gesellschaft. Berlin 2018, Textsortenbeschreibung im Klappentext.

63 Ebd., S. 8.

64 Ebd., S. 7.

65 Ebd., S. 8.

66 Ebd., S. 9. 
spätestens nach ihrer Auflösung klar - genuin kontrafaktischer Elemente: Auf Basis kollektiv gewusster historischer Fakten wie der Präsidentschaft Donald Trumps und der statistisch nachgewiesenen Beliebtheit Mark Zuckerbergs wird eine fiktionale Abweichung vom Geschichtsverlauf erzählt. ${ }^{67}$ Der Präsidentschaftsanspruch und -erfolg des Gurus der digitalen Welt markiert den „Diversionspunkt“, ${ }^{68}$ an dem die Geschichte ihren imaginativ veränderten Verlauf nimmt. Das Kontrafaktische spielt hier ganz elementar mit dem unterschwelligen Wissen um die Wirkmacht von Big Data und ihrer ,Strippenzieher', um über die historische Tatsache der Experimente „mit Formen des social engineering und der ,emotionalen Ansteckung “69 durch Facebook und Co. die Dystopie einer emotional kontrollierten Wählerschaft zu erzeugen. ${ }^{70}$

Beim Weiterlesen der Einführung in den bereits hier erkennbar digitalisierungskritisch oder zumindest -skeptisch argumentierenden Text entpuppt sich Simanowskis Einstieg als ein pädagogischer Kniff: Ziel des Buchs ist eine „Didaktik des Schocks““, ${ }^{71}$ mit der Schüler*innen auf Basis anregender spekulativer Zukunftsszenarien an die Reflexion und Diskussion medialer Beeinflussung ihrer Gegenwart herangeführt werden sollen. Mit Stumme Medien legt Simanowski Lehrer*innen und anderen Vertreter*innen in Vermittlungsberufen wärmstens ans Herz, sich der erwiesenen Wirkkraft digitaler Narrative zu bedienen und ihre Gefahr zu bannen, indem diese Wirkkraft Sichtbarkeit erhält. Die „neue Mediendidaktik“ proklamiert die Nutzbarmachung von Verschwörungstheorien und zieht aus dem pragmatischen Argument, dass sie funktionieren, didaktisch-methodische Konsequenzen für einen medienkritischen Unterricht: „Teenager lieben das Spekulative und werden auf Erklärungen drängen. Na-

67 Die Verbindung von Fakt und Fiktion ist das entscheidende Genremerkmal kontrafaktischer Erzählungen und gleichzeitig das Differenzkriterium zur verwandten Gattung des Historischen Romans. Vgl. dazu Karlheinz Steinmüller: „Zukünfte, die nicht Geschichte wurden. Zum Gedankenexperiment in Zukunftsforschung und Geschichtswissenschaft“. In: Michael Salewski (Hg.): Was wäre wenn. Alternativ- und Parallelgeschichte: Brücken zwischen Phantasie und Wirklichkeit. Stuttgart 1999, S. 43-53.

68 Johannes Dillinger: Uchronie. Ungeschriebene Geschichte von der Antike bis zum Steampunk. Paderborn 2015, S. 14.

69 Simanowski: Stumme Medien, S. 9.

70 Kontrafaktische Erzählungen wie diese sind dann auch nur sinnvoll mit kompositionalistischen Ansätzen zu untersuchen, die nicht von der generellen Fiktionalität aller erhobenen Tatsachen ausgehen, sondern präzise nach den Kombinationen und Umbrüchen von Fakt und Fiktion suchen. Vgl. dazu grundlegend Peter Blume: Fiktion und Weltwissen. Der Beitrag nichtfiktionaler Konzepte zur Sinnkonstitution fiktionaler Erzählliteratur. Berlin 2004 sowie Eva-Maria Konrad: Dimensionen der Fiktionalität. Analyse eines Grundbegriffs der Literaturwissenschaft. Münster 2014.

71 Simanowski: Stumme Medien, S. 23. 
türlich muss man ihnen nicht erst sagen, was Oculus Rift ist oder dass VR für Virtual Reality steht. Aber wie man damit Präsident wird, das werden sie wissen wollen.“72 Simanowski schreibt hier nicht nur ein programmatisches Plädoyer für resignifizierende Verfahren, sondern führt sie mit seinem Text selbst durch, stellt doch der Buchanfang performativ eine didaktische Situation her, in der die Lesenden durch kontrafaktische Spekulation zur Reflexion medialer Einflussnahme angeregt werden sollen. Beschreibung, Programmatik und Vermittlung fallen zusammen und wiederholen die attestierten Eigenschaften des Digitalen als Nährboden für Fake News und Verschwörungstheorien, um mittels Fake News und Verschwörungstheorien Lerneffekte zu erzielen.

Diese durchaus bedenkliche angeleitete Versetzung des eigenen Lektüreprozesses in eine didaktisch-asymmetrische Situation, in der man dem Autor durch Lesen auf den Leim gehen soll, führt damit performativ vor, wie Verfahren der Resignifizierung funktionieren: Die Digitalisierungskritik verfährt selbstreferentiell und unter Zuhilfenahme eines geteilten Kontingenzbewusstseins, um in der Wiederholung des Kritisierten ein eigenes Programm zu etablieren. Der Widerstand Simanowskis, der sich durch Verweise auf Adorno und die Kulturindustrie sowie durch eine an Gumbrecht erinnernde Bezeichnung vermittlungsprädestinierter Personen als „Digital Immigrants“ (im Gegensatz zu „Digital Natives“) selbst als kulturkonservativ markiert, ${ }^{73}$ imitiert somit die Gefahren digitaler Spekulation, um das Gefahrenpotenzial durch Immunisierung der Jugend entlarvend zu neutralisieren und noch dazu eine Anleitung für unterhaltenden Unterricht zu konzipieren. Die kontrafaktische Narration des Beginns wird dazu wiederholt in Form von rhetorischen Fragen aufgerufen und verstetigt; die Digitalisierungskritik macht also den spekulativen Gestus in der strukturellen Konstanz zum prägenden Verfahren ihrer Textgenese - natürlich nicht ohne auch dem lesenden und hoffentlich selbst lehrenden Adressat*innenkreis ebendieses didaktische Verfahren anzuempfehlen.

\section{Daniel Kehlmann: $R$ uhm}

Formal ganz anders, aber mit programmatischen Berührungen und ebenfalls unter Zielsetzung der Resignifizierung verfährt Daniel Kehlmanns vielbeachteter, aus neun Geschichten zusammengesetzter Roman Ruhm (2009). Ruhm ist als literarisches Feuerwerk an autor- und metafiktionalen, intertextuellen sowie

72 Ebd., S. 24.

73 Ebd., S. 13, Herv. i. O. 
surreal-formexperimentellen Verweisen zu bezeichnen. Auf dieser Basis entwirft der Roman mit jeder der neun Geschichten und also insgesamt eine kommunikationstechnologische Reflexion über die gegenwärtige Disposition, mit der Welt verbunden und trotzdem vollkommen isoliert zu schreiben und $\mathrm{zu}$ existieren. Die Metaebene der Einzelnarrationen zeigt die größtenteils kontingente Vernetzung der Figuren untereinander, die auch in der Textperformanz Einfluss aufeinander ausüben, ohne sich wirklich zu begegnen. Kehlmanns Text imitiert somit in Mikro- und Makrostruktur ein Verfahren digitaler Netzrelationen, um eine eigene Poetologie zu skizzieren: Diese verbleibt nicht auf der Ebene digitaler Affirmation oder Kritik, sondern macht sich das Spektrum der Netzmetaphorik zunutze, um an den Möglichkeiten der Weltendarstellung auch die Möglichkeiten des Weltenbewohnens zu verhandeln.

Für die hier zentrale Verfahrenskategorie der Resignifizierung wirft der Text die sprachpragmatisch fundamentale Frage auf, ob Formen der Ironie ebenfalls als subversive Umkehr des Gesagten fungieren können. Kann die Wiederholung im Modus des Uneigentlichen zusätzlich zur bloßen Dekonstruktion der Referenz auch eine produktive Umdeutung leisten? „Ein Beitrag zur Debatte“, die siebte Geschichte im Text, ist ein einziger Forenbeitrag aus Sicht eines zum Klischeeinformatiker stilisierten Erzählers, von dem man an dieser Stelle nur den Usernamen „mollwitt“ erfährt. Die Narration ist über einzelne Figuren und Handlungsstränge mit der ersten und mit der nachfolgenden Geschichte im Roman verbunden; in der Lektüre aller drei Geschichten decken sich schrittweise und nach dem Muster analytischer Erzählungen, wie sie etwa im Kriminalgenre verbreitet sind, erklärende Interdependenzen auf, die vormals nicht entwirrbare Zufälle im Nachhinein kausallogisch motivieren.

Das handlungstragende Moment der ersten Geschichte, „Stimmen“, liegt beispielsweise in der für Betroffene und Telefongesellschaft unerklärlichen Doppelvergabe von Handynummern, die erst in Geschichte acht aufgeklärt, in Geschichte eins jedoch in ihrer Konsequenz erläutert wird: einem kaum sozial vernetzten Durchschnittsbürger auf einmal die - in erster Linie weiblichen Kontaktanfragen an einen bekannten Schauspieler eingebracht, damit sein reales Leben auf den Kopf gestellt und seine Aufmerksamkeit gänzlich ins Virtuelle gezogen zu haben. Verursacher dieser zufälligen Fehlleitung von Kontakten ist besagter „riesen Hardcore-Fan von diesem Forum“ mollwitt aus Geschichte sieben, ${ }^{74}$ der insgesamt trotz seines Berufs nicht nur als lebens- sondern auch als technikunfähig beschrieben und noch dazu sprachlich destabilisiert wird:

74 Daniel Kehlmann: Ruhm. Ein Roman in neun Geschichten. Reinbek bei Hamburg ${ }^{40} 2020$, S. 133. 
Ich weiß, bin zu busy, zu viel Work und Alltag, aber große Thoughts erkenn ich, wenn ich sie sehe. Dann abgelenkt, weil lordoftheflakes den üblichen Bullshit und sich auch proctor, 3helgoland und birnenfreund auf seine Seite geschlagen hatten, dazu zwei Neuposter, die ich gar nicht kannte und denen ich erst mal heavy eins drüberslashen mußte. ${ }^{75}$

Slang, Anglizismen, abgebrochene und dadurch grammatisch-unvollständige Satzteile, Personenbeschreibung nach Nicknames und pointenlose Beiläufigkeit der Sprache ahmen nicht nur die konzeptionelle Mündlichkeit der Kommunikationsform ,Forenbeitrag' nach, sondern parodieren auch den tippenden Geek, der nicht zur Identifikation einlädt, sondern der Lächerlichkeit preisgegeben wird. ${ }^{76}$

Eben dieser lächerliche Protagonist, der im „Real Life (dem wirklichen!)“77 wiederholt „,schwitzend wie immer, beschwert von seinem grotesken Körperumfang, kleingewachsen, nackenlos, bedauernswert“ auftritt, ${ }^{78}$ löst demnach - aus einer den Einzelgeschichten übergeordneten Perspektive betrachtet einen Schmetterlingseffekt aus, der nicht nur das Leben eines völlig Fremden, sondern dadurch auch das des verwechselten Schauspielers und noch dazu das seines Chefs beeinflusst, der wiederum sein Doppelleben zwischen zwei Frauen und zwei Familien mit der Ausrede seines unfähigen Mitarbeiters organisiert. Diese Narration der reigenhaften Zufallskette ${ }^{79}$ konstituiert sich elementar aus resignifizierenden Zitaten des Digitaljargons. Der Jargon steht nicht für sich, sondern dient in dieser ironischen Wiederholung auf den ersten Blick der Figurencharakterisierung, bei genauerem Hinsehen und Weiterlesen der rückwirkenden und vorausweisenden Handlungskonstitution und auf einer weiteren Ebene darüber letztlich der Romanpoetik Kehlmanns, die wiederum über die metafiktionalen Aussagen im Text - „Geschichten in Geschichten in Geschichten. Man weiß nie, wo eine endet und eine andere beginnt“ ${ }^{80}$ - auch Aussagen über Möglichkeiten des Weltkontakts in der Gegenwart treffen. ${ }^{81}$

75 Ebd., S. 140.

76 Vgl. dazu auch Bruno Dupont: „Erzählen im Zeitalter des Internets. Daniel Kehlmanns Ruhm und Daniel Glattauers Gut gegen Nordwind“. In: Germanica 55 (2014), S. 189-207, hier S. 204.

77 Kehlmann: Ruhm, S. 134.

78 Ebd., S. 170.

79 Vgl. Michael Haase: „Die Vernetzung der Welt - zu Daniel Kehlmanns Ruhm“. In: Convivium (2011), S. 345-367, hier S. 353.

80 Kehlmann: Ruhm, S. 201.

81 Die Parallele zwischen Gattungspoetik und Weltkonstruktion sieht auch Kirsten von Hagen: ,,Jeder ist überall, niemand irgendwo“ - Weltwahrnehmung und -konstruktion bei Daniel Kelhmann (Ruhm, 2009) und Giulio Minghini (Fake, 2009)“. In: Christian Moser/Linda Simonis (Hg.): Figuren des Globalen. Weltbezug und Welterzeugung in Literatur, Kunst und Medien. Göttingen 2014, S. 509-521, bes. S. 510 f. 
Die digitalisierungsskeptische Parodie des digital Einheimischen mollwitt nutzt das netzwerksprachliche Zitat, um die Möglichkeiten der Weltordnung überspitzt zu problematisieren. Die Resignifizierung dient hier der textlogisch höhergelagerten Frage nach einer Struktur der gegenwärtigen (literarischen) Weltsituation. Die kritische Nuance des Romans lässt sich damit kaum mehr vom affirmativ-utopischen Gebrauch einer digitalen Ästhetik unterscheiden, nutzt doch auch Kehlmann sie, um das Formrepertoire zu erweitern und besonders avancierte textuelle Verflechtungen zu erzeugen. Die Resignifizierung blendet dann auch die ,Raumversetzung، von Körpern im Digitalen und Fiktionalen ineinander und schafft damit die dominante poetologische These des Romans: „Wenn einer so viel im Internet unterwegs ist wie ich, dann weiß er, daß - wie soll ichs sagen? Also dann weiß er, daß Wirklichkeit nicht alles ist. Daß es Räume gibt, in die man nicht mit dem Körper geht.“"82 Die unablässige Zirkulation von Informationen und Kontaktaufnahmen, die so gut wie nie bei den eigentlich adressierten Figuren ankommen, erschafft eine Poetik fiktiver Zustände, in der virtuell/fiktiv/imaginär ein äquivalentes und zu ergänzendes Paradigma der Uneigentlichkeit bildet, das Stellung nimmt zu Möglichkeiten der Textkonstruktion und darüber hinaus die Lebensbedingungen des 21. Jahrhunderts listet. Der Literatur kommt in diesem Konglomerat an Uneigentlichkeit dann auch keine herausgehobene Position zu, sie wird nicht als das Medium der Kohärenz inmitten von Medien der Vernetzung gerahmt. ${ }^{83}$ Bei Kehlmann dominieren keine Textverfahren der Distanzierung, vielmehr muss sich Literatur in die Bedingungen der globalisierten und kommunikationstechnisierten Gegenwart einschreiben und die Vernetzung der Welt für die Vernetzung des Texts produktiv machen.

Dabei mahnt Ruhm auch nicht, sondern nimmt den Schreibpraktiken im Netz durch Ironie ihr Gefahrenpotenzial. Anders als Austin verlagern Derrida und Butler die Gelingensbedingungen des sprachlichen Ausdrucks nicht in den Kontext, sondern in die Wiederholbarkeit und Wiedererkennbarkeit des Zeichens. Sind bei Austin ästhetische Rahmung und Ironie parasitäre Formen des Sprachrituals, ${ }^{84}$ so verhält sich ihre Wirkung für Derrida nicht nachgelagert. Uneigentliche Ver-

82 Kehlmann: Ruhm, S. 146.

83 Ich stelle mich hier gegen das emphatische Schlusswort Duponts, der ein engagiertes Verständnis von Literatur an den Tag legt, in dem der geformte Text den neuen Medien eine Ordnung geben soll. Vgl. Dupont: „Erzählen im Zeitalter des Internets“, S. 207. Eine solche Kunstemphase ,gegen“ die digitalisierte Welt kann ich in Ruhm nicht erkennen.

84 John L. Austin: Zur Theorie der Sprechakte. Deutsche Bearbeitung v. Eike v. Savigny, 2. Aufl., Stuttgart 1979, S. 44. 
wendungen sind somit trotzdem als Zitat erkennbar und entwickeln aus der performativen Kraft der Zeichen ein transformatives Potenzial. ${ }^{85}$ Von ähnlichen Prämissen geht auch Butler aus, wenn sie dem Ausdruck trotz kontextuell prekärer Bedingungen eine Veränderbarkeit des Kontexts zuschreibt. ${ }^{86}$ Die komische Darstellung mollwitts sowie seines Sprach- und Schriftgebrauchs im Netz nimmt der Netzwelt ihre Ernsthaftigkeit und damit auch ihre Bedrohlichkeit. Auf groteske Usernamen wie „birnenfreund“ oder „ruebendaddy“ reduziert ${ }^{87}$ und durch mollwitts Identität in der realen Welt zusätzlich destabilisiert, sind die Netzakteur*innen eindeutig unsouverän markiert und durch die ironische Resignifizierung in ihrer Handlungsmacht beschnitten. Dass die komisch-groteske Geschichte „Ein Beitrag zur Debatte“ jedoch strukturell von den sehr ernsten und tragischen Geschichten „Stimmen“ und „Wie ich log und starb“ flankiert wird, nimmt der Ironie die Reinheit ihrer Komik. Mollwitts tatsächliche Auswirkungen und die rein zufällig zerstörten Leben der von ihnen bedingten Figuren verleihen der Lektüre der ironischen Uneigentlichkeit einen bitteren Beigeschmack. An der umkehrenden Zielrichtung der Resignifizierung in umgeleitete poetologisch-existenzielle Fragestellungen der Zeit ändert das jedoch nichts.

\section{Ausblick}

Angesichts solcher formal-struktureller Eingeständnisse bleibt die Frage, was die Digitalisierungskritik von der -affirmation unterscheidet, wenn selbst die pejorative Rahmung auf Netzästhetiken zurückgreift. Insgesamt liegt die Vermutung nahe, dass sich die intellektuellen Stimmen unserer Gegenwart in stabilen ideologiekritischen Traditionen bewegen und entsprechende Erklärungsgewohnheiten ausgebildet haben, die den medialen Bedingungen der Diskurspartizipation eine wirklichkeitsstiftende Energie beimessen. Anstatt sich von den beklagten Phänomenen abzuwenden, ist es daher nur konsequent, das Beklagte der Wahrnehmung anheimzustellen und es aus der Gewöhnung in die kritische Reflexion zu transportieren.

85 Vgl. Derrida: „Signatur Ereignis Kontext“, S. 339.

86 Siehe dazu ausführlich Judith Butler: Anmerkungen zu einer performativen Theorie der Versammlung. Übers. v. Frank Born. Berlin 2016. Zwar geht es Butler hier besonders um die Ausdrucksfähigkeit des Körpers; diese leitet sie jedoch von der sprachlichen ab, das in der Performanz subversive Einwirken auf den Kontext gilt also für beide Ausdrucksdimensionen.

87 Kehlmann: Ruhm, S. 145. 
Netzstrukturen erweisen sich darüber hinaus als geeignete Beschreibungskategorie, um aus zeitdiagnostischer Nahsicht Ordnungen zu sehen, die jedoch nur lose verbunden sind und vielmehr assoziativ als essentiell zusammenhängen. Der Objektbereich deckt sich hier also praktisch mit der Perspektive der Beobachtung: Wo der Gegenwartskommentar durch seine Nähe zum Gegenstand zwangsläufig Gefahr läuft, entscheidende Linien und Tendenzen zu übersehen, ist das Netzwerk eine mehr als passable Denkfigur, die Verknüpfungen und Unschärfe integriert - und sich zudem bestens als Anhaltspunkt der Kritik ebendieses Losen und Unscharfen eignet.

Außerdem macht sich bemerkbar, dass auch im digitalkritischen Lager eine Einsicht in die Einflüsse vom Materialen des Medialen auf das Mentale herrscht, ${ }^{88}$ so dass die Gegnerschaft gegen digitale Formen auch mit eben solchen Materialisierungen bestritten werden muss. In mahnenden und resignifizierenden Texten richtet sich die Netzwerkstruktur gegen die Netzwerkstruktur, in distanzierenden werden Lücken des Netzes oder seine lineare Auflösung in Aussicht gestellt. Die Kritik macht demnach überwiegend Formeingeständnisse und unterscheidet sich schließlich durch ihre Pragmatik von der Affirmation. Sie setzt mit der Sichtbarmachung, Alternativgestaltung oder Umkehr Strategien formaler Agency gegen die eingestandene Formdominanz des Digitalen und bemüht sich um überwindende Aussichten aus dem Sog der netzwerkstrukturellen Gleichzeitigkeit.

88 Vgl. dazu einschlägig und überblickshaft Andreas Reckwitz: „Die Materialisierung der Kultur“. In: Friederike Elias u. a. (Hg.): Praxeologie. Beiträge zur interdisziplinären Reichweite praxistheoretischer Ansätze in den Geistes- und Sozialwissenschaften. Berlin/Boston 2014, S. 13-25. 